\title{
Dochead dossier
}

Sous-dochead Les syndromes de Cushing

\section{Les traitements du syndrome Cushing}

\section{Marie-Astrid Beaudoin*}

Praticien hospitalier

\section{Franck Schillo}

Praticien hospitalier, chef de service

Service d'endocrinologie, Centre hospitalier régional universitaire de Besançon, 3 boulevard AlexandreFleming, 25030 Besançon, France

\section{*Auteur correspondant.}

Adresse e-mail : mabeaudoin@chu-besancon.fr (M.-A. Beaudoin).

\section{Résumé}

Les différents traitements d'un syndrome de Cushing permettent de limiter son retentissement. Chirurgie, radiothérapie, traitements médicamenteux agissant sur la surrénale ou sur l'hypophyse, le choix de l'option thérapeutique dépend du profil du patient.
(C) 2019

Mots clés - anticortisolique ; hypophyse ; surrénale ; syndrome de Cushing

Summary à venir

(c) 2019

Keywords à venir 
Le syndrome de Cushing est un hypercorticisme endogène, pouvant être d'origine centrale (adrénocorticotrophine [ACTH]-dépendant) ou périphérique (ACTH-indépendant). Il est à l'origine d'une surmortalité importante, proportionnelle à I'hypercorticisme, principalement cardiovasculaire.

Nous disposons de plusieurs options thérapeutiques, dépendantes de l'étiologie. La chirurgie est le traitement de référence, mais il existe des médicaments ciblant la production d'ACTH, la production de cortisol ou la liaison du cortisol à ses récepteurs, qui peuvent être utilisés en attendant l'intervention, mais aussi en cas de contre-indication ou d'échec de la chirurgie. Ces molécules ont des mécanismes d'action et des profils d'efficacité et de tolérance différents. Il est possible de les associer pour avoir une meilleure efficacité ou améliorer la tolérance. Les objectifs du traitement sont, d'une part, l'exérèse de la tumeur responsable le cas échéant et, d'autre part, le contrôle de l'hypercorticisme.

\section{T1 La chirurgie}

TEG1 La chirurgie hypophysaire par voie transsphénoïdale réalisée par un opérateur entrainé est le traitement de première intention de la maladie de Cushing, lorsque l'exérèse complète de l'adénome est envisageable [1,2]. Elle permet de contrôler l'hypersécrétion dans 65 à $90 \%$ des micro-adénomes et dans moins de $65 \%$ des macro-adénomes. Le risque de récidive, qui peut survenir jusqu'à des dizaines d'années après l'intervention initiale, s'établit entre 20 et $25 \%$ [2-4]. Finalement, 40 à $50 \%$ des patients requièrent un traitement supplémentaire [5]. La rémission est obtenue lorsque le patient présente une insuffisance corticotrope, il nécessite donc une supplémentation glucocorticoïde.

TEG1 Une surrénalectomie unilatérale est proposée dans les situations suivantes :

- adénome surrénalien unilatéral avec extinction de l'autre surrénale à la scintigraphie au noriodocholestérol ;

- corticosurrénalome unilatéral ;

- certains cas d'hyperplasie macronodulaire (HMN) bilatérale des surrénales mais asymétrique [1].

En cas d'HMN, le risque de récidive sur la seconde surrénale est important, mais la chirurgie unilatérale permet de retarder la surrénalectomie bilatérale et ses comorbidités.

TEG1 Une surrénalectomie bilatérale peut être envisagée en présence d'HMN bilatérale des surrénales, d'hyperplasie micronodulaire ou de désir de grossesse à court terme dans la maladie de Cushing [1]. Celle-ci permet de corriger l'hyperproduction cortisolique, au prix d'une double substitution (gluco- et minéralocorticoïde) par la suite avec, cependant, un risque de décompensation bien connu des insuffisances surrénaliennes. Si l'hypercorticisme est sévère, la surrénalectomie bilatérale peut être réalisée en urgence, comme traitement de sauvetage. La qualité de vie paraît être moins bonne chez les patients ayant une maladie de Cushing traitée par surrénalectomie bilatérale que chez ceux ayant bénéficié d'une chirurgie hypophysaire, d'une radiothérapie et/ou d'un traitement anti-cortisolique [6]. 
TEG1 En cas de syndrome de Cushing secondaire à une sécrétion ectopique d'ACTH par une tumeur neuro-endocrine, le plus souvent pulmonaire, le traitement de référence est l'exérèse complète de cette tumeur, lorsqu'elle est localisée par les examens d'imagerie.

\section{T1 La radiothérapie hypophysaire}

TEG1 Proposée dans les maladies de Cushing non guéries par la chirurgie ou non opérées, la radiothérapie hypophysaire est un traitement de seconde ou troisième intention, particulièrement indiqué dans les tumeurs agressives ou invasives.

TEG1 La radiothérapie stéréotaxique est la méthode de référence. Elle permet de délivrer une grosse dose de radiations au niveau de la tumeur en limitant l'atteinte des tissus sains adjacents. Elle peut être réalisée en une seule fois (radiochirurgie stéréotaxique, par Gamma Knife ou protonthérapie) ou en plusieurs temps (radiothérapie stéréotaxique fractionnée) [7]. L'efficacité est en moyenne de 50 à $75 \%$ sur l'hypersécrétion hormonale, avec une action retardée jusqu'à 16 mois, et de 90 \% sur le contrôle du volume tumoral [7]. Les complications sont l'hypopituitarisme, les troubles neurocognitifs, les accidents cérébro-vasculaires et les processus néoplasiques [7].

\section{T1 Les traitements médicamenteux anticortisoliques}

TEG1 Les molécules anticortisoliques bloquent la production surrénalienne de cortisol et sont principalement utilisées dans les syndromes de Cushing ACTH-dépendants, en cas d'impossibilité (complication aiguë, contre-indication) ou d'échec de la chirurgie, en traitement préopératoire, en attendant les effets de la radiothérapie, mais aussi parfois dans les syndromes de Cushing ACTHindépendants [1].

TEG1 L'évaluation de l'efficacité se fait sur les mesures répétées du cortisol libre urinaire des 24 heures (CLU). L'objectif principal est d'obtenir sa normalisation, dans les valeurs basses de la norme, c'est-à-dire comprise entre 10 et $20 \mu \mathrm{g} / 24 \mathrm{~h}$. Le rétablissement d'un cycle nycthéméral normal de production cortisolique est très difficile et sera l'objectif secondaire après normalisation du CLU.

TEG1 Le kétoconazole est un dérivé imidazolé, initialement utilisé comme antifongique, qui inhibe la majorité des enzymes en jeu dans la production de cortisol (11- $\beta$-hydroxylase, 17- $\alpha$-hydroxylase, 17,20 lyase). Il en résulte une diminution franche de la production cortisolique et une accumulation des précurseurs précoces dans la stéroïdogenèse : la progestérone et la 17-OH progestérone (figure 1, encadré 1). De plus, une action inhibitrice au niveau pituitaire est suspectée devant l'absence ou la faible élévation de l'ACTH en réaction à la baisse du cortisol sous traitement, dans le cadre d'une maladie de Cushing [8].

En raison d'une très grande rapidité d'action du kétoconazole (48 heures), la première évaluation du CLU est effectuée au bout d'une semaine. Ce médicament pouvant entraîner une insuffisance 
surrénalienne, la surveillance du cortisol à huit heures est nécessaire, avec un premier dosage trois jours après l'introduction.

La dose initiale est choisie en fonction de la sévérité de l'hypercorticisme : 400 à $600 \mathrm{mg} / \mathrm{jour}$ en cas d'hypercorticisme modéré (CLU < $200 \mu \mathrm{g} / 24 \mathrm{~h}$ ) et jusqu'à $1200 \mathrm{mg} /$ jour en présence d'hypercorticisme sévère (CLU > $200 \mu \mathrm{g} / 24 \mathrm{~h}$ ), en trois prises. À terme, ce traitement permet de diminuer le taux de cortisol chez 30 à $80 \%$ des patients, une normalisation étant obtenue chez $50 \%$ d'entre eux $[3,4,9]$.

\section{Encadré 1. À surveiller sous kétoconazole}

Du fait de l'augmentation de la progestérone et de la diminution de la production de testostérone par le blocage enzymatique, le kétoconazole entraîne des gynécomasties (13 \%) et des hypogonadismes périphériques, ce qui en fait plutôt un traitement de seconde intention chez I'homme [4]. De rares cas d'hépatites médicamenteuses ont été décrits (2,5\% des patients traités) [9]. II est donc nécessaire de surveiller le bilan hépatique, particulièrement dans les six premiers mois. Cependant, une augmentation modérée transitoire des transaminases peut être observée en début de traitement, par induction enzymatique, et n'est pas prédictive de la survenue d'une hépatite [10]. Enfin, le kétoconazole a une action inhibitrice sur les cytochromes P450, il faut donc surveiller les paramètres liés aux autres traitements pris par le patient.

Sur une colonne près de son appel

TEG1 La métyrapone est un puissant inhibiteur de la 11- $\beta$-hydroxylase, enzyme clé de la stéroïdogenèse. Il en résulte une accumulation des précurseurs, le 11-désoxy-cortisol et la désoxycorticostérone (DOC) (figure 1, encadré 2), ainsi qu'une augmentation des androgènes : androstènedione, $17-\mathrm{OH}$ progestérone et testostérone, par redirection des précurseurs vers la voie des androgènes qui reste la seule voie qui n'est pas bloquée.

L'action de la métyrapone est très rapide : le cortisol commence à baisser deux heures après la première administration [11]. Sa demi-vie étant courte, quatre prises par jour sont conseillées. La dose initiale est dépendante du degré d'hypercorticisme : $3000 \mathrm{mg} /$ jour en cas d'hypercorticisme modéré et jusqu'à 6000 mg/jour dans les hypercorticismes sévères, en trois ou quatre prises. Elle sera ensuite adaptée à la réponse biologique. Le traitement est efficace sur le contrôle de I'hypercorticisme chez 75 à $83 \%$ des patients souffrant d'une maladie de Cushing, $81 \%$ de ceux ayant un adénome ou un carcinome surrénalien et $70 \%$ de ceux présentant une sécrétion ectopique d'ACTH, avec des doses plus importantes [11].

Le dosage du cortisol plasmatique sous métyrapone est difficile. En effet, la majorité des dosages de cortisol croisent avec le 11-désoxycortisol, qui est fortement augmenté. Le cortisol plasmatique peut alors être faussement normal alors que le patient se trouve en insuffisance surrénalienne.

La surveillance prend donc uniquement en compte le CLU, après vérification auprès du laboratoire de l'absence de réaction croisée avec les précurseurs. 
Il existe un risque d'insuffisance surrénalienne (13\%) [4], mais en raison de l'augmentation de la DOC, qui a une activité minéralocorticoïde, aucune supplémentation en minéralocorticoïdes n'est nécessaire. L'hydrocortisone doit être introduite lors de la baisse trop importante du CLU.

\section{Encadré 2. À surveiller sous métyrapone}

L'augmentation de la désoxycorticostérone (DOC) sous métyrapone est rapide et importante. Elle favorise l'hypertension artérielle et l'hypokaliémie par son activité minéralocorticoïde, ainsi que des malaises, entraînés par son métabolite, la tétra-hydro-DOC. L'augmentation des androgènes est à l'origine d'un hirsutisme, ce qui en fait plutôt un traitement de seconde intention chez la femme. L'ajout de kétoconazole bloque la 17- $\alpha$-hydroxylase, donc la voie des androgènes, et permet de limiter l'hirsutisme.

Sur une colonne près de son appel

TEG1 Le mitotane, initialement utilisé comme insecticide, est un médicament adrénolytique à action lente qui pénètre dans les cellules corticosurrénaliennes grâce au cholestérol et entraîne une destruction mitochondriale aboutissant à la mort cellulaire. II a également un effet direct inhibiteur sur les enzymes précoces de la stéroïdogenèse (11- $\beta$-hydroxylase, 3- $\beta$-hydroxystéroïde déshydrogénase). De plus, il entraîne une surexpression du cytochrome 3A4 (CYP3A4), qui réduit la biodisponibilité du cortisol [2].

L'effet escompté est tardif, après trois mois d'utilisation. Les fonctions corticosurrénaliennes diminuent progressivement ; la zone réticulée (androgènes) est atteinte en premier, puis la zone fasciculée (glucocorticoïdes) et la zone glomérulée (minéralocorticoïdes).

Le traitement est débuté à la dose de $500 \mathrm{mg} / \mathrm{jour}$, puis augmenté assez rapidement jusqu'à 3000 à $5000 \mathrm{mg} / \mathrm{jour}$, en trois prises. L'objectif dans le syndrome de Cushing est de bloquer la production cortisolique surrénalienne. L'adaptation se réalise en fonction du CLU, puisque le cortisol plasmatique est faussé par la diminution de sa biodisponibilité et l'augmentation de la cortisol binding globulin (CBG) (encadré 3). En l'absence de seuil minimal efficace, la mitotanémie est uniquement dosée pour éviter le surdosage. Le mitotane permet une normalisation du CLU dans $80 \%$ des cas, mais $60 \%$ des patients rechuteront à l'arrêt du traitement, ce qui montre que l'effet adrénolytique n'est pas le principal mode d'action [2,12]. Une substitution gluco- et minéralocorticoïde est nécessaire. L'autorisation de mise sur le marché (AMM) française du mitotane concerne uniquement le carcinome corticosurrénalien.

\section{Encadré 3. À surveiller sous mitotane}

Le mitotane est un inducteur enzymatique puissant au niveau hépatique. Cet effet est plus rapide que la baisse du cortisol, de l'ordre de quatre semaines. Cela entraîne l'augmentation de la cortisol 
binding globulin (CBG) et l'accélération du métabolisme de nombreux médicaments, dont les glucocorticoïdes. La dose d'hydrocortisone nécessaire pour substituer l'insuffisance surrénalienne sera donc nettement augmentée.

Une contraception efficace est nécessaire chez la femme, pendant au moins cinq ans après la fin du traitement, du fait de l'effet rémanent du mitotane [13]. II est préférable d'éviter la pilule estroprogestative dans ce contexte d'induction enzymatique.

Sur une colonne près de son appel

TEG1 L'osilodrostat est un puissant inhibiteur de la 11- $\beta$-hydroxylase de longue durée d'action, en cours d'étude. Il entraîne la baisse de plus de 50 \% du CLU chez tous les patients ainsi qu'une normalisation chez 80 à $92 \%$ d'entre eux. Son efficacité est plus importante que celle de la métyrapone. La dose conseillée varie de 4 à $100 \mathrm{mg} /$ jour, en une prise [14,15].

\section{Encadré 4. À surveiller sous osilodrostat}

L'osilodrostat entraîne une augmentation du 11-désoxycortisol, à l'origine des mêmes problèmes d'interaction dans le dosage du cortisol et des mêmes effets secondaires que la métyrapone.

Sur une colonne près de son appel

TEG1 L'étomidate, anesthésiant général, est un inhibiteur de la 11- $\beta$-hydroxylase, avec un effet dosedépendant. C'est le seul traitement anti-cortisolique disponible par voie intraveineuse. La posologie proposée est un bolus de $0,03 \mathrm{mg} / \mathrm{kg}$ suivi d'une perfusion de 0,03 à $0,3 \mathrm{mg} / \mathrm{kg} /$ heure. L'effet, observé à partir de cinq heures après l'administration, est maximal 11 heures après [4].

\section{T1 Les substances agissant sur la sécrétion d'ACTH}

TEG1 La cabergoline est un agoniste des D2R utilisé dans le traitement de l'hyperprolactinémie. Au niveau hypophysaire, la dopamine a une action inhibitrice sur la production de prolactine. Elle agit par les récepteurs dopaminergiques de type 2 et 4 (D2R et D4R) qui sont exprimés au niveau de I'antéhypophyse. Les D2R sont également exprimés sur $80 \%$ des adénomes corticotropes et sur certaines tumeurs neuro-endocrines bien différenciées produisant de l'ACTH [16].

L'action de la cabergoline sur la diminution de la sécrétion d'ACTH est modeste, mais peut permettre une amélioration de la maladie de Cushing. Les études montrent environ $30 \%$ de réponse persistante après trois ans de traitement à raison de $2 \mathrm{mg} /$ semaine $[17,18]$. La dose initiale est de 0,5 à $1 \mathrm{mg} / \mathrm{semaine}$, elle est progressivement augmentée jusqu'à un maximum de $6 \mathrm{mg} / \mathrm{semaine}$. L'AMM française ne concerne que l'hyperprolactinémie. 
TEG1 Le pasiréotide est un analogue de la somatostatine ayant une action prédominante sur les SSTR5 (encadré 5). La majorité des adénomes corticotropes ( $85 \%$ ) expriment des récepteurs à la somatostatine de type 2 et 5 (SSTR2 et SSTR5). L'activité des SSTR2 est diminuée par l'excès de cortisol, contrairement à celle des SSTR5 $[2,19]$.

In vitro, le pasiréotide permet une diminution de la production d'ACTH de 23 à $56 \%$ et inhibe la prolifération cellulaire de 10 à $70 \%$ [20]. In vivo, il favorise une normalisation du CLU chez $40 \%$ des patients $[21,22]$, une réduction du volume tumoral et une baisse de I'ACTH. L'efficacité varie selon les sujets, puisque l'expression des SSTR5 n'est pas similaire selon les adénomes. Les patients répondeurs ou non peuvent être identifiés après deux mois de traitement $[4,21]$.

Le pasiréotide était initialement développé en injections sous-cutanées biquotidiennes. Il existe maintenant une forme à libération prolongée, en injection intramusculaire mensuelle, de 10 à $40 \mathrm{mg}$, dont l'efficacité est identique à celle de la forme à libération immédiate [22]. L'AMM française concerne la maladie de Cushing.

\section{Encadré 5. À surveiller sous pasiréotide}

Le pasiréotide induit de fréquents troubles de la glycorégulation (50 à $73 \%$ ), voire des diabètes, par diminution de la production de glucagon like peptide-1 (GLP-1) et baisse de l'insulinosécrétion.

Sur une colonne près de son appel

\section{T1 Les antagonistes des récepteurs aux glucocorticoïdes}

TEG1 La mifépristone a une affinité importante pour les récepteurs aux glucocorticoïdes avec un très faible effet agoniste. Elle permet donc de bloquer l'effet des glucocorticoïdes avec, en contrepartie, une augmentation de l'ACTH et du cortisol plasmatiques (encadré 6).

Une nette amélioration du retentissement de l'hypercorticisme est observée chez plus de $80 \%$ des patients [4,5,23]. La dose est de 300 à $1200 \mathrm{mg} /$ jour. L'évaluation de l'efficacité est difficile, puisqu'il n'y a aucun marqueur en dehors de l'amélioration du retentissement de l'hypercorticisme [4].

Cette molécule est peu utilisée en France puisqu'elle n'a pas l'AMM pour l'hypercorticisme, mais elle est approuvée par la Food and Drug Administration pour le traitement du diabète chez les patients atteints de syndrome de Cushing. 


\section{Encadré 6. À surveiller sous mifépristone}

Les principaux effets secondaires de la mifépristone sont l'hypokaliémie et l'augmentation de l'épaisseur endométriale [23]. Les effets sur la taille de l'adénome hypophysaire et sur l'endomètre à long terme ne sont pas connus [4].

Sur une colonne près de son appel

\section{T1 Les combinaisons de traitements}

TEG1 Dans le syndrome de Cushing modéré, une combinaison de traitements peut permettre une action centrale et périphérique ou des mécanismes d'action différents, pour mieux contrôler le CLU en limitant les effets secondaires.

Une réponse complète est obtenue dans $53 \%$ des cas avec un traitement par pasiréotide et cabergoline et dans $88 \%$ des cas, avec l'association pasiréotide, cabergoline et kétoconazole [24]. L'ajout de kétoconazole à petite dose ( $\leq 400 \mathrm{mg} /$ jour) permet de normaliser le CLU chez $66 \%$ des patients insuffisamment contrôlés par la cabergoline seule, tout en limitant les effets secondaires [18].

Un traitement combiné par kétoconazole et métyrapone à petite dose permet une meilleure efficacité en améliorant la pharmacodynamie et la couverture du nycthémère, tout en limitant les effets secondaires.

TEG1 Dans le syndrome de Cushing sévère, le contrôle de l'hypercorticisme devient une urgence. Une association de kétoconazole et métyrapone à forte dose permet une action rapide et importante : la CLU atteint une valeur 16 à 40 fois supérieure à la normale diminue jusqu'à 1 à 3,2 fois cette même valeur en une semaine [25]. L'ajout de mitotane dès le début du traitement peut permettre un sevrage des autres molécules après trois mois. La tolérance reste bonne et la trithérapie apparaît comme une alternative à la surrénalectomie bilatérale de sauvetage [26].

\section{T1 Conclusion}

Le syndrome de Cushing est une maladie rare et sévère, dont la prise en charge peut s'avérer compliquée. Il existe plusieurs options thérapeutiques dont chacune offre une efficacité et un profil de tolérance différents.

En dehors des situations d'urgence, il est primordial d'analyser tous les éléments cliniques, biologiques et radiographiques avant de prendre une décision thérapeutique, d'où la nécessité d'une prise en charge personnalisée, après discussion en réunion multidisciplinaire ainsi qu'avec le patient.

Points à retenir 
- L'exérèse totale de l'adénome hypophysaire par chirurgie est le traitement de première intention dans la maladie de Cushing.

- La radiothérapie sert en seconde intention en cas d'échec de la chirurgie ou en présence d'une tumeur agressive ou invasive.

- La surrénalectomie bilatérale n'est proposée qu'en dernière intention et nécessite une substitution en minéralo- et glucocorticoïdes.

- Les traitements médicamenteux sont utilisés en cas de contre-indication ou d'échec de la chirurgie, en attente des effets de la radiothérapie et comprennent : les anticortisoliques, les molécules inhibant la sécrétion d'adrénocorticotrophine (ACTH) et les antagonistes des récepteurs aux glucocorticoïdes.

Sur une colonne après la puce de fin

\section{Références}

[1] Haute Autorité de santé (HAS). Syndrome de Cushing. Protocole national de diagnostic et de soins. Septembre 2008. www.has-sante.fr/portail/upload/docs/application/pdf/2008-

12/pnds_syndrome_de_cushing_version_web_051208.pdf

[2] Cuevas-Ramos D, Fleseriu M. Treatment of Cushing's disease: a mechanistic update. J Endocrinol. 2014;223(2):R19-39.

[3] Castinetti F, Morange I, Jaquet P et al. Ketoconazole revisited: a preoperative or postoperative treatment in Cushing's disease. Eur J Endocrinol. 2008;158(1):91-9.

[4] Fleseriu M, Petersenn S. Medical management of Cushing's disease: what is the future? Pituitary. 2012;15(3):330-41.

[5] Fleseriu M. Medical management of persistent and recurrent Cushing disease. Neurosurg Clin N Am. 2012;23(4):653-68.

[6] Sarkis P, Rabilloud M, Lifante JC et al. Bilateral adrenalectomy in Cushing's disease: Altered longterm quality of life compared to other treatment options. Ann Endocrinol. 2018;500034266(18)30019-2.

[7] Pivonello R, De Leo M, Cozzolino A et al. The Treatment of Cushing's Disease. Endocr Rev. 2015;36(4):385-486.

[8] Tabarin A, Navarranne A, Guérin J et al. Use of ketoconazole in the treatment of Cushing's disease and ectopic ACTH syndrome. Clin Endocrinol. (Oxf.) 1991;34(1):63-9.

[9] Castinetti F, Guignat L, Giraud P et al. Ketoconazole in Cushing's disease: is it worth a try? J Clin Endocrinol Metab. 2014;99(5):1623-30. 
[10] Young J, Bertherat J, Vantyghem MC et al; Compassionalte use Programme. Hepatic safety of ketoconazole in Cushing's syndrome: results of a Compassionate Use Programme in France. Eur J Endocrinol. 2018;178(5):447-58.

[11] Verhelst JA, Trainer PJ, Howlett TA et al. Short and long-term responses to metyrapone in the medical management of 91 patients with Cushing's syndrome. Clin Endocrinol. (Oxf.) 1991;35(2):16978.

[12] Luton JP, Mahoudeau JA, Bouchard P et al. Treatment of Cushing's disease by O,p'DDD. Survey of 62 cases. N Engl J Med. 1979;300(9):459-64.

[13] Leiba S, Weinstein R, Shindel B et al. The protracted effect of o, $p^{\prime}-D D D$ in Cushing's disease and its impact on adrenal morphogenesis of young human embryo. Ann Endocrinol. 1989;50(1):49-53.

[14] Bertagna X, Pivonello R, Fleseriu M et al. LCl699, a potent 11ß-hydroxylase inhibitor, normalizes urinary cortisol in patients with Cushing's disease: results from a multicenter, proof-of-concept study. J Clin Endocrinol Metab. 2014;99(4):1375-83.

[15] Fleseriu M, Pivonello R, Young J et al. Osilodrostat, a potent oral 11 $\beta$-hydroxylase inhibitor: 22week, prospective, Phase II study in Cushing's disease. Pituitary. 2016;19(2):138-48.

[16] Gatto F, Hofland LJ. The role of somatostatin and dopamine D2 receptors in endocrine tumors. Endocr Relat Cancer. 2011;18(6):R233-51.

[17] Godbout A, Manavela M, Danilowicz K et al. Cabergoline monotherapy in the long-term treatment of Cushing's disease. Eur J Endocrinol. 2010;163(5):709-16.

[18] Vilar L, Naves LA, Azevedo MF et al. Effectiveness of cabergoline in monotherapy and combined with ketoconazole in the management of Cushing's disease. Pituitary. 2010;13(2):123-9.

[19] de Bruin C, Feelders RA, Lamberts SWJ et al. Somatostatin and dopamine receptors as targets for medical treatment of Cushing's Syndrome. Rev Endocr Metab Disord. 2009;10(2):91-102.

[20] Batista DL, Zhang X, Gejman R et al. The effects of SOM230 on cell proliferation and adrenocorticotropin secretion in human corticotroph pituitary adenomas. J Clin Endocrinol Metab. 2006;91(11):4482-8.

[21] Colao A, Petersenn S, Newell-Price J et al; Pasireotide B2305 Study Group. A 12-month phase 3 study of pasireotide in Cushing's disease. N Engl J Med. 2012;366(10):914-24.

[22] Lacroix A, Gu F, Gallardo W et al; Pasireotide G2304 Study Group. Efficacy and safety of oncemonthly pasireotide in Cushing's disease: a 12 month clinical trial. Lancet Diabetes Endocrinol. 2018;6(1):17-26.

[23] Fleseriu M, Biller BMK, Findling JW; SEISMIC Study Investigators. Mifepristone, a glucocorticoid receptor antagonist, produces clinical and metabolic benefits in patients with Cushing's syndrome. J Clin Endocrinol Metab. 2012;97(6):2039-49. 
[24] Feelders RA, de Bruin C, Pereira AM et al. Pasireotide alone or with cabergoline and ketoconazole in Cushing's disease. N Engl J Med. 2010;362(19):1846-8.

[25] Corcuff JB, Young J, Masquefa-Giraud P et al. Rapid control of severe neoplastic hypercortisolism with metyrapone and ketoconazole. Eur J Endocrinol. 2015;172(4):473-81.

[26] Kamenický P, Droumaguet C, Salenave S et al. Mitotane, metyrapone, and ketoconazole combination therapy as an alternative to rescue adrenalectomy for severe ACTH-

dependent Cushing's syndrome. J Clin Endocrinol Metab. 2011;96(9):2796-804.

Déclaration de liens d'intérêts

Les auteurs déclarent ne pas avoir de liens d'intérêts.

Figure

Beau_fig1

Figure 1. La stéroïdogenèse surrénalienne.

(C) MA. Beaudoin

Sur deux colonnes près de son appel 